\title{
Research on Logistic Management Quality Evaluation Based on Support Vector Machine
}

\author{
Ya-qing Deng ${ }^{a}$, Shuang $\mathrm{Yu}^{\mathrm{b}}$ and Ning Wang ${ }^{\mathrm{c}}$ \\ Commercial Technology Department,Baoding Vocational and Technical College,Baoding,China \\ a4871028@qq.com, b1807969989 @qq.com, '262104794@qq.com
}

\begin{abstract}
Keywords: Logistics management; Quality evaluation; Support vector machine
\end{abstract}
Abstract. For making feasible logistics management come true, fully using data resources, and establishing in line with the development of a healthy enterprise logistics management mode, this paper puts forward a new analysis model for evaluating the quality level of logistics management based on support vector machine theory. By analyzing the influence factors in the logistics business process, the proposed model predicts management efficiency of logistics operations implementation, and provide theoretical support for logistics management system optimization. The support vector machine is of accuracy and efficiency, which is effective for the quality evaluation of logistics management.

\section{Introduction}

In the development and construction of logistics information system, the existing logistics system design approaches are usually based on work flow mode that the business process designing is to personal management experience, the development cycle is shorter, the impact of the success of logistics and cost savings are not fully considered. These defects result in the original data can not fully used in evaluation and predicting of the logistics activities, and the data are wasteful [1,2]. It is big problem need to be solved that how to fully inspire the parties involved in the logistics information system, analyze the various risk factors, correctly evaluate the influence of various factors, establish the quality evaluation model of logistics system management and achieve the maximum benefit [3]. Support vector machine is a sophisticated and highly principled learning method for designing a sub-linear element only contained in the hidden layer of back forward network. SVM is based on the risk minimization principle of VC dimension theory. It can overcome many shortcomings of traditional methods and neural network model. The prior knowledge of specific issues are not required. In the case of limited training samples, it can effectively control the machine learning ability [4]. Thus, it can be effectively utilized for multi-dimensional quality evaluation of logistics management [5].

\section{Establishment of Logistics management quality evaluation index system}

Through the logistics business implementation process, the main objects involves logistics enterprises and staff in each sectors. Due to the implementation of the logistics business is not done by a single person, but collaboration is done by multiple logistic personnel, so only set a post evaluation when considering the impact of human factors. Thus, logistics management quality evaluation index system in the designing process contains two major factor: the logistics enterprises evaluation index system and logistics individual evaluation system, which is shown as follows:

Basic information. Storage quality $\left(\mathrm{X}_{1}\right)$, The number of loading and unloading equipment $\left(\mathrm{X}_{2}\right)$, Freight vehicle mass $\left(\mathrm{X}_{3}\right)$, The average age of employees employed $\left(\mathrm{X}_{4}\right)$, The proportion of college education $\left(\mathrm{X}_{5}\right)$. 
Human factors. The driver working years $\left(\mathrm{X}_{6}\right)$, The number of traffic violations $\left(\mathrm{X}_{7}\right)$, Traffic accident rate $\left(\mathrm{X}_{8}\right)$, The number of road congestion $\left(\mathrm{X}_{9}\right)$, Service attitude $\left(\mathrm{X}_{10}\right)$.

Logistics quality. Distribution correct rate $\left(\mathrm{X}_{11}\right)$, On-time delivery rate $\left(\mathrm{X}_{12}\right)$, Commodity good rates $\left(\mathrm{X}_{13}\right)$, Packaging intact rate $\left(\mathrm{X}_{14}\right)$, Back to a single complete rate $\left(\mathrm{X}_{15}\right)$.

Service quality. Remind inspection rate $\left(\mathrm{X}_{16}\right)$, Promise to honor the rate $\left(\mathrm{X}_{17}\right)$, Customer complaint rate $\left(\mathrm{X}_{18}\right)$, Credit scores $\left(\mathrm{X}_{19}\right)$, Service innovation rate $\left(\mathrm{X}_{20}\right)$.

\section{Support vector machine}

Support vector machine is a sophisticated and highly principled learning method for designing a sub-linear element only contained in the hidden layer of back forward network. SVM is based on the risk minimization principle of $\mathrm{VC}$ dimension theory. It can overcome many shortcomings of traditional methods and neural network model. The prior knowledge of specific issues are not required. In the case of limited training samples, it can effectively control the machine learning ability.

Considering a two classification models, a training sample is defined as $\left\{\left(x_{i}, y_{i}\right)\right\}$, where $x_{i}$ represents the input vector and $y_{i}$ represents the classification sign. The two classes of linear discriminant function of separable cases are as follows:

$$
f(x)=w \cdot \varphi(x)+b
$$

Where $x$ is the sample vector, $w$ is the weight vector, $b$ represents the classification threshold.

Supposing there is a classification decision plane:

$$
f(x)=w \cdot \varphi(x)+b=0 .
$$

Which satisfies:

$$
f(x)=\left\{\begin{array}{ll}
w \cdot \varphi(x)+b>0 & y_{i}=+1 \\
w \cdot \varphi(x)+b<0 & y_{i}=-1
\end{array} .\right.
$$

Thus, Eq.(2) is defined as the classification and ultra flat surface of support vector.

A two types of linear separable linear sample set $\left\{\left(x_{i}, y_{i}\right)\right\}^{N}, i=1,2, \cdots, n$ is given. The weight vector $w$ in hyperplane and classification threshold $b$ are both obtained. As to any support vector $x_{s v}$ and its classification label $y_{s v}$, it satisfies the following condition.

$$
f\left(x_{s v}\right)=W^{T} x_{s v}+b=\left\{\begin{array}{ll}
+1 & y_{s v}=+1 \\
-1 & y_{s v}=-1
\end{array} .\right.
$$

The maximum interval of the classification plane can be calculated as $2 /\|w\|$, Therefore, the maximum class interval is equivalent to the minimum. If the surplus plane need to classify all samples correctly, it must satisfy:

$$
y_{i}\left(W^{T} x_{i}+b\right)-1 \geq 0, i=0,1,2, \cdots, N \text {. }
$$


Thus, the SVM hyperplane by solving the following constrained optimization problem was the solution:

$$
\min _{w} \frac{1}{2} W^{T} W
$$

Which is a A typical quadratic programming problem. The optimal solution is a saddle point of the following Lagrangian:

$$
L(w, b, a)=\frac{1}{2}(w, w)^{T}-\sum_{i=1}^{l} \alpha_{i}\left[\left(x_{i} w-b\right)-1\right] .
$$

Where $a_{i}$ is the Lagrange multipliers, The above optimization problem inverts into a dual form to get the solution:

$$
\max W(\alpha)=\min \left(-\frac{1}{2} \sum_{i, j=1}^{n} \alpha_{i} \alpha_{j} y_{i} u_{j} k\left(x_{i}, x_{j}\right)\right) \sum_{i=1}^{n} \alpha_{i} .
$$

According KKT conditions, those points on the boundary of two types of sample points fall in support vector machine SVM required solution. Classification decision function is obtained as follows:

$$
f(x)=\operatorname{sign}\left(\sum_{i, j=1}^{n} \alpha_{i} y_{i} k\left(x_{i}, x_{j}\right)+b\right) .
$$

Where $k\left(x_{i}, x_{j}\right)$ is the kernel function.

The Gaussian kernel function is selected as the support vector machine kernel function in this paper. The form of Gaussian kernel function is shown as follows:

$$
K(x, y)=\exp \left(-\frac{\|x-y\|^{2}}{\sigma^{2}}\right)
$$

Thus, the optimal separating hyperplane is required:

$$
f(x)=\operatorname{sign}\left(\sum_{i, j=1}^{n} \alpha_{i} y_{i} \exp \left(-\frac{\left\|x_{i}-x_{j}\right\|^{2}}{\sigma^{2}}\right)+b\right) .
$$

For SVM with Gaussian kernel function, the parameters conclude penalty parameter $C$ and kernel parameter $\sigma$. The penalty parameter $C$ is made between the structure and the risk of sample error compromise. Kernel width $\sigma$ is related to the input space of learning sample or the width. 


\section{Case study}

Some logistics management information system data of a certain company is selected as a sample in this paper. There are total 150 samples after sorting. The former 140 samples are regarded as the training samples, the remaining 10 samples are regarded as the testing samples.

\section{The selection of parameters in SVM.}

First of all, keep penalty parameter $C$ as 10 , gradually adjust the value of kernel parameter $\sigma$ until the mean square error (MSE) of the overall test is minimum, the optimal kernel parameter $\sigma^{*}$ is obtained. Then, in the same way, keep $\sigma$ as $\sigma^{*}$, gradually adjust the value of $C$ until the mean square error (MSE) of the overall test is minimum, the optimal penalty parameter $C^{*}$ is obtained. The fitness function in the adjustment process and the various steps is selected as MSE. MSE is defined as follows:

$$
M S E=\sqrt{\sum_{i=1}^{n} \frac{\left(y^{*}-y_{i}\right)^{2}}{n}} .
$$

Where $y_{i}$ is the actual value and $y^{*}$ is the prediction value.

The selection process of parameters is shown in Table 1.

Table 1. Parameters selection of SVM

\begin{tabular}{|c|c|c|c|}
\hline \multicolumn{2}{|c|}{$C=10$} & \multicolumn{2}{c|}{$\sigma=20$} \\
\hline$\sigma$ & MSE & $C$ & MSE \\
\hline 0.1 & 0.9245 & 1 & $1.12487393 \mathrm{e}-5$ \\
\hline 0.5 & 0.8749 & 30 & $1.12428236 \mathrm{e}-5$ \\
\hline 0.9 & 0.5612 & 70 & $1.12398213 \mathrm{e}-5$ \\
\hline 1.0 & 0.2108 & 80 & $1.12398209 \mathrm{e}-5$ \\
\hline 8.0 & 0.0038 & 82 & $1.12398162 \mathrm{e}-5$ \\
\hline 12.0 & $1.2175 \mathrm{e}-4$ & 83 & $1.12398134 \mathrm{e}-5$ \\
\hline 16.0 & $8.2417 \mathrm{e}-5$ & 84 & $1.12398120 \mathrm{e}-5$ \\
\hline 20.0 & $2.1231 \mathrm{e}-5$ & 89 & $1.12398136 \mathrm{e}-5$ \\
\hline 30.0 & $4.1290 \mathrm{e}-5$ & 800 & $6.17923212 \mathrm{e}-4$ \\
\hline 38.0 & $8.5356 \mathrm{e}-4$ & 1500 & 0.00781919 \\
\hline 42.0 & $9.9609 \mathrm{e}-4$ & 2000 & 0.00812939 \\
\hline 50.0 & $4.1243 \mathrm{e}-3$ & 2500 & 0.01798102 \\
\hline
\end{tabular}

It can be seen from Table 1, when $C$ is kept as 10, $\sigma$ takes 20, which can make the MSE minimum. When $\sigma$ is kept as 20,C takes 86 , which can make the MSE minimum. Therefore, the optimal penalty parameter $C$ is 86 , and the optimal kernel parameter $\sigma$ is 20 .

\section{Training and testing.}

The former140 samples of the total 150 samples are regarded as the training samples. The optimal parameters selected above are put into SVM. Through the training process, the result shows that the number of support vector is 113 , the value of $b$ is 0.0002376 . Thus, the support vector regression model is shown as follows:

$$
f(x)=\sum_{i=1}\left(a_{i}-a_{i}^{*}\right)\left(x \cdot x_{i}+1\right)+0.0002376
$$


Put the remaining 10 testing sample in the trained SVM, and the test results are shown in Table 2.

Table 2. Test results of SVM

\begin{tabular}{|c|c|c|c|}
\hline Testing sample & Actual value & Output value of SVM & Relative error [\%] \\
\hline 141 & 0.67 & 0.671 & 0.19 \\
\hline 142 & 0.28 & 0.279 & 0.50 \\
\hline 143 & 0.47 & 0.478 & 0.34 \\
\hline 144 & 0.52 & 0.511 & 0.81 \\
\hline 145 & 0.35 & 0.355 & 0.91 \\
\hline 146 & 0.29 & 0.288 & 0.15 \\
\hline 147 & 0.77 & 0.777 & 0.41 \\
\hline 148 & 0.66 & 0.659 & 0.16 \\
\hline 149 & 0.54 & 0.537 & 0.85 \\
\hline 150 & 0.24 & 0.245 & 0.24 \\
\hline
\end{tabular}

It can be seen from Table 2, the largest relative error in testing sample is $0.91 \%$, the lowest relative error in testing sample is $0.16 \%$, and the average relative error is $0.46 \%$. Each testing sample's relative is below $1 \%$, which indicates that the proposed model has a good generalization. Therefore, SVM model can be applied to evaluate the quality of logistics information management system, and provide the decision-making basis for optimization of logistics information management system.

\section{Conclusions}

Through processing the data of logistics system, the quality evaluation indexes of logistics management are obtained. According to the training sample set, the quality evaluation of logistics management model based on support vector machine is established. The influence of single effective logistics activities from four sub- index systems including basic situation of the company, human factors, affecting the quality of logistics, and quality of service can be analyzed. The sub-index systems also have their lower index decomposition, which form the twenty secondary indicators as the key research targets. The test error of SVM is low, and SVM has a high efficiency, which can be applied to practice, evaluate the quality level of logistics management and provide a theoretical basis for decision support development system.

\section{References}

[1] Hisao Fujimoto. Strategic Management for Environmental Logistics Channel. Procedia-Social and Behavioral Sciences. Vol. 58 (2012), p. 1443-1447.

[2] Zoran Miodrag, Christan Tesch, Uwe Clausen. Improving Resource Management of Large Logistics Facilities. Procedia-Social and Behavioral Sciences. Vol. 54 (2012), p. 12-18.

[3] Slavica Dožić, Milica Kalić. An AHP Approach to Aircraft Selection Process. Transportation Research Procedia. Vol. 3 (2014), p. 165-174.

[4] F. Lolli, A. Ishizaka, R. Gamberini. New AHP-based approaches for multi-criteria inventory classification. International Journal of Production Economics. Vol. 156 (2014), p. 62-74.

[5] Ajay Kumar, Ravi Shankar, Roma Mitra Debnath. Analyzing customer preference and measuring relative efficiency in telecom sector: A hybrid fuzzy AHP/DEA study. Telematics and Informatics. Vol. 32 (2015), p. 447-462. 